\title{
Effective treatment of severe acute pancreatitis and COVID-19 pneumonia with tocilizumab
}

\author{
Piotr Zielecki ${ }^{1}$, Magdalena Kaniewska ${ }^{1}$, Mariusz Furmanek ${ }^{2}$, Tomasz Bulski², Grażyna Rydzewska ${ }^{1,3}$ \\ ${ }^{1}$ Clinical Department of Internal Medicine and Gastroenterology with Inflammatory Bowel Disease Unit, Central Clinical \\ Hospital of the Ministry of the Interior and Administration, Warsaw, Poland \\ ${ }^{2}$ Department of Radiology, Central Clinical Hospital of the Ministry of the Interior and Administration, Warsaw, Poland \\ ${ }^{3}$ Collegium Medicum, Jan Kochanowski University, Kielce, Poland
}

Gastroenterology Rev 2020; 15 (3): 267-272

DOI: https://doi.org/10.5114/pg.2020.99042

\begin{abstract}
Address for correspondence: Piotr Zielecki, Clinical Department of Internal Medicine and Gastroenterology with Inflammatory Bowe Disease Unit, Central Clinical Hospital of the Ministry of the Interior and Administration, 137 Wołoska St, 02-507 Warsaw, Poland, e-mail: piotrzielecki@gmail.com
\end{abstract}

In December 2019 the coronavirus disease 2019 (COVID-19) caused by Severe Acute Respiratory Syndrome Coronavirus 2 (SARS-CoV-2) was reported for the first time in Wuhan, China [1], and it subsequently rapidly spread to the rest of the world. On $11^{\text {th }}$ March 2020 COVID-19 was declared a pandemic by the World Health Organisation [2]. The most common symptoms are fever, cough, shortness of breath, fatigue, myalgia, and loss of smell and taste [3], but gastrointestinal symptoms like vomiting, diarrhoea, and abdominal pain are also reported $[4,5]$. SARS-CoV-2 uses angiotensin-converting enzyme (ACE) 2 as an entry receptor to infect host cells [6]. The highest ACE 2 expression was found in alveolar cells of the lungs [7], but also in the heart, kidneys, and gastrointestinal tract, including the pancreas. To date, it is not known if SARS-CoV-2 can cause pancreatic cell damage leading to acute pancreatitis, but in many cases serum lipase and amylase levels are elevated [8]. A study also showed that patients with history of acute pancreatitis may be more susceptible to COVID-19, but the mechanism of this phenomenon is not known yet [9]. Many severe COVID-19 patients develop acute respiratory distress syndrome (ARDS), which is the most serious complication of SARS-CoV-2 infection [10]. It has been reported that a systemic inflammatory syndrome called cytokine release syndrome (CRS) is responsible for the development of ARDS in SARS-CoV-2 infection, which leads to pulmonary fibrosis and organ failure. In this pathophysiological process interleukin-6 (IL-6), and B and T cells play key roles [11]. Tocilizumab (TCZ) is a monoclonal antibody that competitively inhibits the binding of IL-6 to its receptor (IL-6R). This mechanism blocks receptor complex signal transduction to inflammatory mediators responsible for $B$ and $T$ cell activation and inhibits $c y$ tokine storm [12]. IL-6 pathway blocking can be a new method for the treatment of severe COVID-19 patients [13], and tocilizumab is expected to become an effective drug against COVID-19, but currently scientific data are limited. One study showed that TCZ might reduce mortality in patients with severe COVID-19 pneumonia [14], but to date there have been no human studies evaluating tocilizumab in the treatment of acute pancreatitis. U.S. Food and Drug Association (FDA)-approved phase III randomised controlled trials on tocilizumab are ongoing [15]. We report the first (to date) case study of a patient with severe acute pancreatitis and COVID-19 pneumonia treated effectively with tocilizumab.

On April 4, 2020 a 38-year-old male with late oedematous moderate acute pancreatitis, hepatic steatosis, hepatosplenomegaly, and alcohol dependence was admitted to the Central Clinical Hospital of the Interior and administration in Warsaw, Poland (hospital designated for SARS-CoV-2 infected patients only) with positive SARS-CoV-2 molecular test result on $3^{\text {rd April }}$ 2020. He had previously been hospitalised for 2 weeks in another medical centre due to severe acute pancreatitis presenting with acute-onset upper abdominal pain, metabolic acidosis, and features of multi-organ failure. Empiric antimicrobial therapy with vancomycin and imipenem-cilastatin intravenously and total parenteral nutrition (TPN) were administered there. On $27^{\text {th }}$ March 2020 he underwent chest high-resolution computed tomography (HRCT) and abdomen-pelvis contrast-enhanced computed tomography (CECT) scans, which 
showed diffuse enlargement, shaggy contour of the pancreas without contrast enhancement of pancreatic neck, body, and tail, oedema and blurring of peripancreatic fat planes, acute peripancreatic and intraperitoneal fluid collection (Computed Tomography Severity Index (CTSI) -8 points), bilateral atelectasis in lower lung segments, and pleural fluid collections up to $24 \mathrm{~mm}$ on the left side and up to $18 \mathrm{~mm}$ on the right side. No COVID-19 imaging findings were shown. The patient was transferred to our hospital to continue the treatment due to SARS-CoV-2 infection. At admission the patient was in good clinical condition and presented mild shortness of breath without cough or abdominal pain. He did not have a history of contact with SARSCoV-2 infected person. His physical examination revealed fever with body temperature of $38^{\circ} \mathrm{C}$, arterial blood pressure of $150 / 92 \mathrm{~mm} \mathrm{Hg}$, tachycardia of 124 beats per minute, respiratory rate of 20 breaths per minute, and oxygen saturation of $91 \%$ while breathing ambient air. The contour of the abdomen was slightly prominent and distended. There was no abdominal tenderness or rebound pain. No oedema was observed in lower limbs. Physiological reflexes were present. Laboratory tests showed moderate anaemia, hypoalbuminaemia, hypocalcaemia, increased level of C-reactive protein (CRP), aspartate aminotransferase (AST), alanine aminotransferase (ALT), $\gamma$-glutamyl transpeptidase (GGTP), lactate dehydrogenase (LDH), lipase, and platelet (PLT) count (Table I). Venous blood gas analysis (oxygen flow $3 \mathrm{l} / \mathrm{min}$ by nasal catheter) showed no significant abnormalities. After admission the patient received paracetamol $1000 \mathrm{mg}$ four times a day intravenously and oxygen supplementation through a nasal cannula at a flow of $3-4 \mathrm{l} / \mathrm{min}$, and his oxygen saturation increased to $95 \%$. Vancomycin 1000 mg twice a day, meropenem $1000 \mathrm{mg}$ three times a day intravenously, and dalteparin 7500 IU once a day subcutaneously were administered. According to current recommendations of the Hospital's Therapeutic Committee, chloroquine $500 \mathrm{mg}$ orally twice a day was administered. Blood and urine culture tests were negative. The patient was tested for hepatitis B surface antigen, hepatitis $C$ antibodies, and human immunodeficiency virus (HIV) antigen, which were negative. The patient was provided with a mechanical soft diet with good clinical tolerance. On day 2 his vital parameters were stable, and he received oral azithromycin $500 \mathrm{mg}$ daily. On days 3 through 6 of hospitalisation the patient's vital parameters remained stable except fever at $37.5-39.1^{\circ} \mathrm{C}$ (despite paracetamol and metamizole treatment), mild dry cough, and shortness of breath with oxygen saturation at 91-94\% while breathing ambient air. The nasal cannula was exchanged for an oxygen mask at a flow rate of 5-6 l/min.
Laboratory tests performed on days 4 and 5 showed that CRP and procalcitonin (PCT) levels were increased (Table I). On day 3 an abdomen-pelvis CECT scan was performed and compared to previous CECT ( $27^{\text {th }}$ March 2020), which showed necrosis of more than $50 \%$ of pancreatic parenchyma (body and tail), peripancreatic and along Gerota's fascia fluid collections, and acute necrosis collection (ANC) along the greater curvature of the stomach with dimensions of $88 \times 70 \times 125 \mathrm{~mm}-$ CTSI -8 pts (Figures $1 \mathrm{~A}$ and B). On day 5 a chest X-ray was performed and showed diffused bilateral alveolar consolidations (Figure 2). On day 6 abdominal ultrasound and emergency chest HRCT were performed. HRCT showed widespread, multifocal, bilateral groundglass opacities (GGO) typical for COVID-19 pneumonia (Figure $3 \mathrm{~A}$ ) and ultrasound showed hepatic enlargement up to $180 \mathrm{~mm}$, enlargement of pancreas up to $30 \mathrm{~mm}$ and fluid collection $120 \times 60 \mathrm{~mm}$ located around head of pancreas and along the left anterior renal fascia. On the same day vancomycin was discontinued, and the patient received lopinavir/ritonavir $400 \mathrm{mg} / 100 \mathrm{mg}$ orally twice a day. The next day the patient's body temperature was still increased up to $39.4^{\circ} \mathrm{C}$, and CRP and D-dimers levels increased. A serum IL- 6 level test was performed. Later at night, due to persistent fever, increased serum IL-6 level (367 pg/ml), and after multidisciplinary consultation, the patient received tocilizumab (Roche, Basel, Switzerland) 800 mg intravenously to inhibit cytokine storm syndrome. There were no adverse reactions during and after the infusion. On day 8 the patient's condition improved and body temperature was normal. On day 10 CRP level $(33.9 \mathrm{mg} / \mathrm{l})$ dramatically decreased, the patient's oxygen saturation improved to 98\% while breathing ambient air. Meropenem was discontinued, and rifaximin $400 \mathrm{mg}$ orally twice a day was administered. On day 12 abdominal ultrasound showed $112 \times 45 \times 60 \mathrm{~mm}$ peripancreatic fluid collection (as in previous abdominal ultrasound examination), parenchymal heterogenicity of body and tail of pancreas, and spleen enlargement up to $127 \mathrm{~mm}$. On day 13 a nasopharyngeal swab test for SARS-CoV-2 nucleic acid was negative, CRP level (10.7 mg/l) decreased, and red blood cell morphology improved showing elevation of RBC count, and HCT and HGB level (Figure 4). Chloroquine was discontinued. The next day retesting of nasopharyngeal swabs for SARS-CoV-2 nucleic acid was negative and control chest HRCT showed significant regression of GGO and reduction of pleural fluid collection (Figure $3 \mathrm{~B}$ ). On day 15 (18 ${ }^{\text {th }}$ April 2020), due to clinical and laboratory improvement without the need for oxygen supplementation and negative SARS-CoV-2 swabs tests, the patient was discharged from the hospital with advice of undertaking control abdominal ultrasound within 
Table I. Laboratory tests before and after tocilizumab infusion

\begin{tabular}{|c|c|c|c|c|c|c|c|c|}
\hline \multirow[t]{2}{*}{ Parameter } & \multirow[t]{2}{*}{ Normal range } & \multicolumn{4}{|c|}{ Before tocilizumab } & \multicolumn{3}{|c|}{ After tocilizumab } \\
\hline & & $D-5$ & $D-3$ & $\mathrm{D}-2$ & D 0 & D 1 & D 3 & D 6 \\
\hline Albumin $[\mathrm{g} / \mathrm{dl}]$ & $3.5-5.2$ & 2.66 & & & 2.99 & & & \\
\hline Total protein [g/dl] & $6.4-8.3$ & 5.86 & & & 6.81 & & & \\
\hline $\mathrm{ALP}[\mathrm{U} / \mathrm{I}]$ & 40-129 & & & & 97 & 118 & & \\
\hline GGTP [U/I] & $8-61$ & 239 & & & 106 & 128 & & \\
\hline $\mathrm{ALT}[\mathrm{U} / \mathrm{I}]$ & $5-41$ & 85 & & & 43 & 50 & 45 & \\
\hline $\mathrm{AST}[\mathrm{U} / \mathrm{I}]$ & $5-37$ & 103 & & & 34 & 35 & 31 & \\
\hline Total bilirubin [mg/dl] & $0.3-1.2$ & 0.48 & & & 0.41 & 0.42 & & \\
\hline $\mathrm{Ca}^{2+}[\mathrm{mmol} / \mathrm{l}]$ & $2.09-2.54$ & 1.76 & & & & 2.15 & & \\
\hline $\mathrm{K}^{+}[\mathrm{mmol} / \mathrm{l}]$ & $3.5-5.3$ & 4.01 & 4.31 & 4.53 & 4.71 & 4.49 & 4.59 & 4.85 \\
\hline $\mathrm{Na}^{+}[\mathrm{mmol} / \mathrm{l}]$ & $135-145$ & 136 & 139 & 133 & 139 & 139 & 135 & 135 \\
\hline $\mathrm{CRP}[\mathrm{mg} / \mathrm{l}]$ & $0-5$ & 189.4 & 152.8 & 146.4 & 196.6 & 176.2 & 33.9 & 10.7 \\
\hline PCT [ng/ml] & $0.0-0.5$ & 0.51 & 0.71 & 0.58 & & & & \\
\hline D-dimer $[\mu g / l$ FEU] & $0-500$ & & & & 6238 & & 15694 & 24500 \\
\hline Total cholesterol [mg/dl] & 0-190 & 67 & & & & 103 & & \\
\hline Glucose [mg/dl] & 70-99 & & & & 113 & & 91 & \\
\hline Creatinine [mg/dl] & $0.7-1.2$ & 0.69 & 0.64 & 0.62 & 0.62 & 0.64 & 0.65 & 0.94 \\
\hline $\mathrm{EGFR}[\mathrm{ml} / \mathrm{min}]$ & & 128 & 140 & 145 & 145 & 140 & 139 & 90 \\
\hline Urea [mg/dl] & $17-49$ & 15 & 17 & & 20 & & 13 & \\
\hline $\mathrm{LDH}[\mathrm{U} / \mathrm{I}]$ & $135-225$ & 633 & & & 463 & & 443 & 469 \\
\hline Lipase [U/l] & $13-60$ & 133 & & & & & & \\
\hline Amylase [U/I] & $28-100$ & 44 & & & 44 & 49 & & \\
\hline White cell count $\left[\times 10^{9} / 1\right]$ & $4.5-10.0$ & 8.47 & 8.03 & 6.55 & 6.99 & 4.33 & 5.95 & 10.2 \\
\hline Red cell count $\left[\times 10^{12} / 1\right]$ & $4.7-6.1$ & 3.44 & 3.48 & 3.21 & 3.33 & 3.48 & 3.8 & 4.39 \\
\hline Haemoglobin [g/dl] & $14.0-17.0$ & 9.9 & 10 & 9.3 & 9.7 & 9.8 & 10.8 & 12.6 \\
\hline Hematocrit (\%) & $42.0-52.0$ & 30.9 & 32.1 & 29.3 & 30.2 & 31.5 & 34.1 & 38.1 \\
\hline Platelet count $\left[\times 10^{9} / 1\right]$ & $150-400$ & 461 & 583 & 280 & 605 & 640 & 750 & 730 \\
\hline Neutrophil count $\left[\times 10^{9} / 1\right]$ & $1.9-7.0$ & 6.5 & 5.89 & 4.49 & 5.21 & 2.51 & 3.06 & 5.41 \\
\hline Lymphocyte count $\left[\times 10^{9} / I\right]$ & $0.9-5.0$ & 1.21 & 1.29 & 1.35 & 0.98 & 1.16 & 1.86 & 2.99 \\
\hline INR & $0.9-1.2$ & 1.43 & & & 1.47 & 1.42 & 1.36 & 1.27 \\
\hline Prothrombin time [s] & $9.4-12.5$ & 15.9 & & & 16.3 & 15.7 & 15.1 & 14 \\
\hline APTT $[s]$ & $25.4-36.9$ & 31.7 & & & 33.2 & & & \\
\hline
\end{tabular}

$D$ - days before and after tocilizumab infusion. 

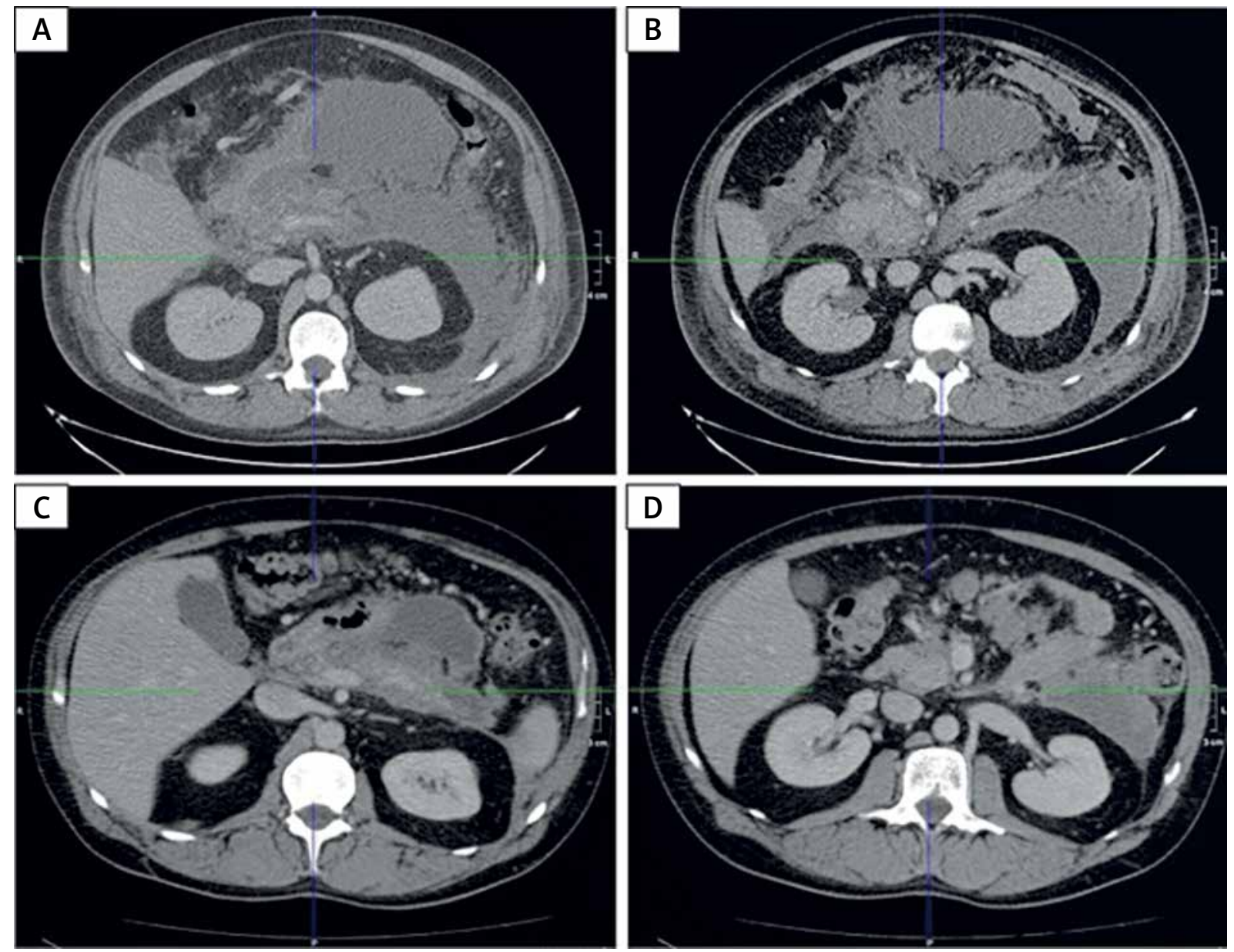

Figure 1. Abdomen and pelvis CECT images on day 3 of hospitalization and 10 weeks after discharge. A, B - CECT images on day 3 show oedema and necrosis of pancreatic parenchyma (body and tail), peripancreatic fluid collections, and acute necrosis collection (ANC) along greater curvature of stomach. C, D - CECT images 10 weeks after discharge shows regression of pancreatic oedema and peripancreatic fluid and evolution of ANC to sterile WON

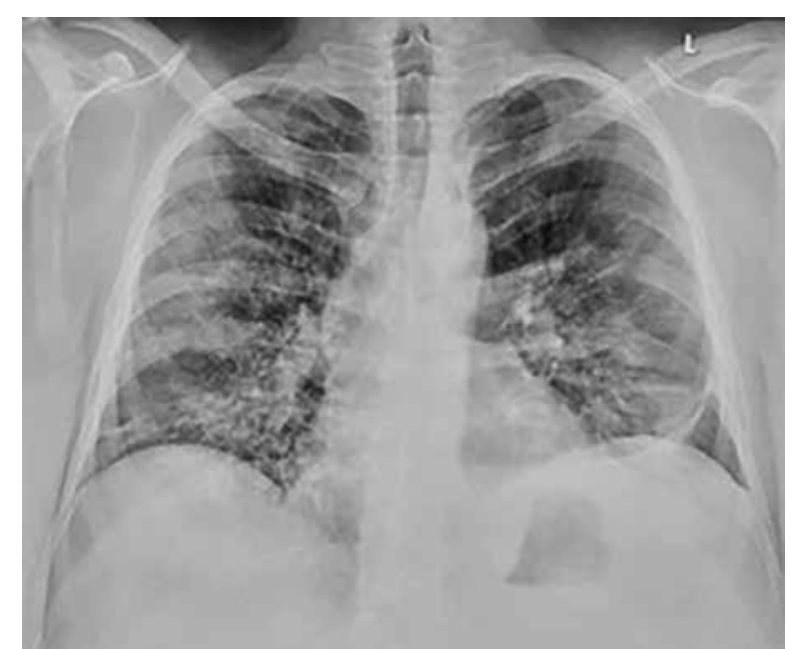

Figure 2. Chest X-ray performed on day 5 showing diffused bilateral alveolar consolidations typical in COVID-19 pneumonia
3-4 weeks, and chest HRCT and blood tests within 2 months. Control ambulatory abdominal ultrasound performed on $3^{\text {rd }}$ June 2020 revealed $130 \times 55 \times 40 \mathrm{~mm}$ inflammatory changes around the head of the pancreas, body and tail heterogenous necrosis, and $130 \times 55 \times 40$ $\mathrm{mm}$ peripancreatic and along anterior renal fascia fluid collection. CRP level $(7.6 \mathrm{mg} / \mathrm{l})$, blood morphology parameters, sodium, potassium, transaminases, ALP, GGTP, bilirubin, amylase, lipase, creatinine, urea, and TSH were within normal range (Table I). On $1^{\text {st }}$ July 2020 control chest HRCT, abdomen-pelvis CECT, and blood tests were performed. Chest HRCT showed no pulmonary consolidations (Figure $3 \mathrm{C}$ ). Abdomen-pelvis CECT revealed regression of pancreatic oedema and peripancreatic fluid collection, necrosis of more than $50 \%$ of pancreatic parenchyma (poor contrast enhancement of body and tail parenchyma as previously), $61 \times 42 \times 38 \mathrm{~mm}$ encapsulated wall-off necrosis (WON) along the greater gastric 

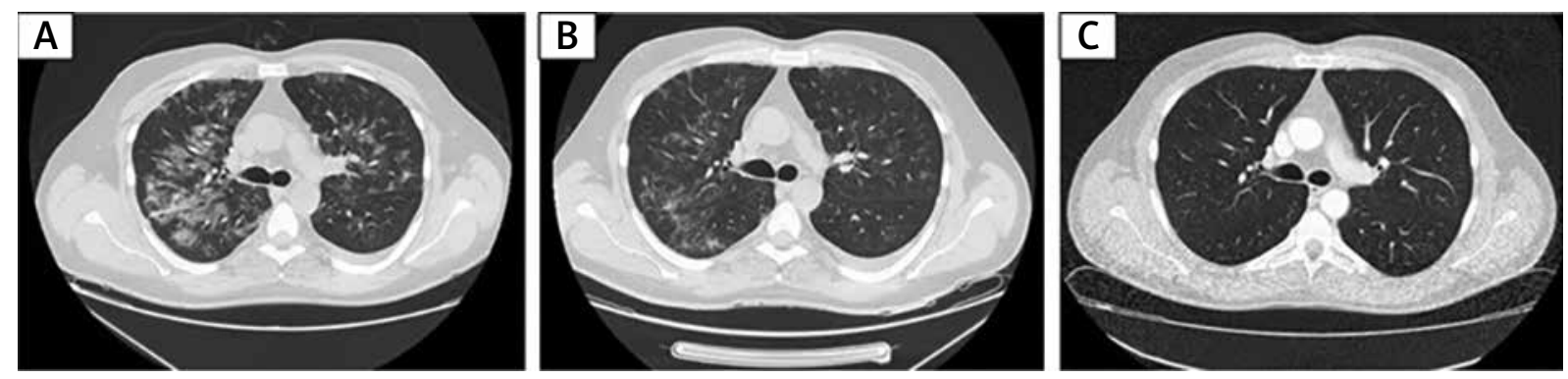

Figure 3. Chest HRCT images on day 6, day 14 of hospitalisation, and 10 weeks after discharge. A - Chest CT image on day 6 shows ground glass subpleural opacities in both lungs typical in COVID-19 pneumonia. B - Chest CT image on day 14 (7 days after tocilizumab dose) shows regression of ground glass opacities. C - Chest CT image 10 weeks after discharge shows no significant abnormalities in both lungs

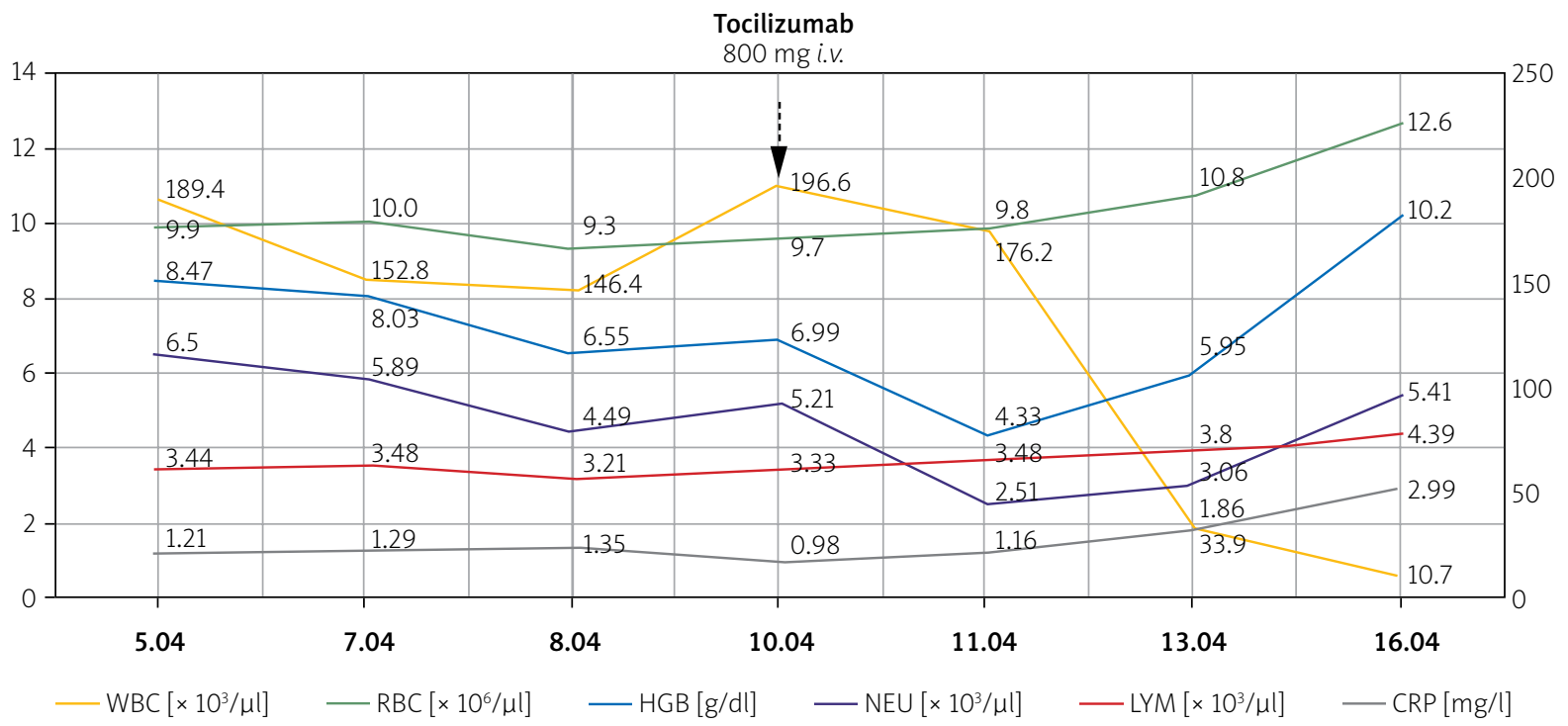

Figure 4. Blood morphology tests and CRP levels before and after tocilizumab infusion show a significant decrease in CRP level, a decrease of WBC count on the first day, and an increase on the second day after infusion and an increase in RBC count and HGB level

curvature (previously ANC $88 \times 70 \times 125 \mathrm{~mm}$ ), encapsulated $78 \times 32 \times 180 \mathrm{~mm}$ WON along the left anterior renal fascia down to the descending colon, and splenic vein thrombosis with collateral vessel compensation (Figures $1 \mathrm{C}$ and D). After hospital discharge the patient experienced only mild abdominal pain periodically a couple of times a month, mainly after dietary mistakes; no other symptoms were reported, and his physical examination showed no significant abnormalities. Outpatient follow-up was continued throughout 2.5 months with good outcome.

Pancreatic injury (described as increased serum amylase and lipase levels) can occur during SARS-CoV-2 infection with a prevalence of COVID-19 greater among patients with a history of acute pancreatitis [9]. It is still not clear whether SARS-CoV-2 can induce viral pancreatic inflammation. We report a 38-year-old male with
COVID-19 pneumonia and severe acute pancreatitis of probably alcoholic aetiology. At admission to the hospital the patient presented with signs and symptoms of acute pancreatitis without typical COVID-19 manifestation. After a couple of days a chest HRCT showed typical COVID-19 findings. The patient's condition deteriorated, he had fever up to $39.4^{\circ} \mathrm{C}$, high CRP and IL-6 levels, and anaemia. There were no evident effects of antiviral medicine, and tocilizumab was administered. Within 3 days after tocilizumab infusion the patient's CRP level and body temperature significantly decreased, his HGB level increased, and after 7 days the pulmonary changes regressed. The patient's condition and respiratory efficiency improved. Control HRCT performed 7 days after tocilizumab infusion showed regression of ground-glass opacities in both lungs. Sterile necrosis of pancreatic parenchyma developed to encapsulated sterile walled-off 
necrosis without further complications. These observations show that tocilizumab might be effective against inflammatory response in COVID-19. The only observed side effect of tocilizumab infusion was a decrease in WBC count on the second day, which is already a well reported side effect. It is difficult to determine the effectiveness of tocilizumab in patients with acute pancreatitis and COVID-19 pneumonia. Studies performed in 2016 and 2017 showed that tocilizumab may be significantly effective in experimental severe acute pancreatitis and acute lung injury in rats models [16, 17]; however, other study showed that pancreatitis may be caused by tocilizumab-induced hypertriglyceridaemia [18]. Data on humans is still limited, and we need more information about the incidence, course, and outcome of acute pancreatitis associated with COVID-19 pneumonia. We report on the first (to date) case study of a patient with COVID-19 pneumonia associated with acute pancreatitis effectively treated with tocilizumab. Understanding the mechanisms of SARS-CoV-2-related pancreatic injury is crucial to create appropriate strategies of therapy in this group of patients.

\section{Conflict of interest}

The authors declare no conflict of interest.

\section{References}

1. Commission WMH. Report of clustering pneumonia of unknown etiology in Wuhan City. Published December 31, 2019. 2020.

2. WHO. WHO Director-General's opening remarks at the media briefing on COVID-19 - 11 March 2020. 2020; https://www. who.int/dg/speeches/detail/whodirector-general-s-openingremarks-at-the-media-briefing-on-covid-19---11-march-2020

3. Fu L, Wang B, Yuan T, et al. Clinical characteristics of coronavirus disease 2019 (COVID-19) in China: a systematic review and meta-analysis. J Infect 2020; 80: 656-65.

4. Xiao F, Tang M, Zheng X, et al. Evidence for gastrointestinal infection of SARS-CoV-2. Gastroenterology 2020; 158: 1831-3. e3.

5. Wong SH, Lui RNS, Sung JJY. Covid-19 and the digestive system. J Gastroenterol Hepatol 2020; 35: 744-8.

6. Zhou P, Yang XL, Wang XG, et al. A pneumonia outbreak associated with a new coronavirus of probable bat origin. Nature 2020; 579: 270-3.

7. Hamming I, Timens W, Bulthuis ML, et al. Tissue distribution of ACE2 protein, the functional receptor for SARS coronavirus. A first step in understanding SARS pathogenesis. J Pathol 2004; 203: 631-7.

8. Liu F, Long X, Zhang B, et al. ACE2 expression in pancreas may cause pancreatic damage after SARS-CoV- 2 infection. Clin Gastroenterol Hepatol 2020; 18: 2128-30.e2.

9. Gubatan J, Levitte S, Patel A, et al. Prevalence, risk factors and clinical outcomes of COVID-19 in patients with a history of pancreatitis in Northern California. Gut 2020. doi: 10.1136/ gutjnl-2020-321772.

10. Yang X, Yu Y, Xu J, et al. Clinical course and outcomes of critically ill patients with SARS-CoV-2 pneumonia in Wuhan, China: a single-centered, retrospective, observational study. Lancet Respir Med 2020; 8: 475-81.

11. Zhou Y, Fu B, Zheng X, et al. Aberrant pathogenic GM-CSF+ $T$ cells and inflammatory CD14+CD16+ monocytes in severe pulmonary syndrome patients of a new coronavirus. BioRxiv 2020; 2020.02.12.945576.

12. Sebba A. Tocilizumab: the first interleukin-6-receptor inhibitor. Am J Health Syst Pharm 2008; 65: 1413-8.

13. Zhang C, Wu Z, Li JW, et al. Cytokine release syndrome in severe COVID-19: interleukin-6 receptor antagonist tocilizumab may be the key to reduce mortality. Int J Antimicrob Agents 2020; 55: 105954.

14. Guaraldi G, Meschiari M et al. Tocilizumab for treatment of patients with severe COVID-19: a retrospective cohort study. Lancet Rheumatol 2020, doi.org/10.1016/S2665-9913(20)30173-9.

15. A Study to Evaluate the Safety and Efficacy of Tocilizumab in Patients With Severe COVID-19 Pneumonia (COVACTA). https://clinicaltrials.gov/ct2/show/NCT04320615.

16. Chen KL, Lv ZY, Yang HW, et al. Effects of tocilizumab on experimental severe acute pancreatitis and associated acute lung injury. Crit Care Med 2016; 44: e664-77.

17. Hançerli Y, Kaplan M, Tanoğlu A, et al. Efficacy of tocilizumab treatment in cerulein-induced experimental acute pancreatitis model in rats. Turk J Gastroenterol 2017; 28: 485-91.

18. Morrison AR, Johnson JM, Ramesh M, et al. Acute hypertriglyceridemia in patients with COVID-19 receiving tocilizumab. J Med Virol 2020. doi: 10.1002/jmv.25907.

Received: 24.08 .2020

Accepted: 24.08 .2020 